Pathologe 2015 · 36:5-6

DOI 10.1007/s00292-014-2028-6

Online publiziert: 30. Januar 2015

(c) Springer-Verlag Berlin Heidelberg 2015
T. Mentzel ${ }^{1}$ P. Schirmacher ${ }^{2}$ E. Bierhoff ${ }^{3}$

${ }^{1}$ Dermatopathologie Bodensee, Friedrichshafen

2 Pathologisches Institut, Universitätsklinikum Heidelberg

${ }^{3}$ Heinz-Werner-Seifert-Institut für Dermatopathologie, Bonn

\title{
Neoplasien der Haut
}

Sehr geehrte Leserinnen und Leser,

die Haut ist das größte menschliche Organ und diversen schädigenden Faktoren, u. a. dem UV-Licht ausgesetzt. Neoplasien der Haut, insbesondere Karzinome und maligne Melanome, haben in den letzten Jahren teilweise dramatisch zugenommen. Ursache hierfür sind u. a. das erhöhte Lebensalter und die auch damit zusammenhängende, zunehmende aktinische Schädigung der Haut mit ihren Folgen. So erkrankten nach einer Hochrechnung des Krebsregisters SchleswigHolstein in Deutschland im Jahre 2010 etwa 234.400 Personen neu; die Tendenz ist weiter steigend. Darüber hinaus existiert seit Juli 2008 in Deutschland eine Früherkennungsuntersuchung auf Hautkrebs; die gesetzlich Versicherten haben ab dem 35. Lebensjahr alle 2 Jahre Anspruch auf eine derartige Untersuchung. Diese Tatsachen und der Umstand, dass einige dermatologische Einrichtungen, die früher selbst histologische Untersuchungen durchgeführt haben, dies aber aktuell aus verschiedenen Gründen nicht mehr praktizieren, haben bei den Pathologen in Deutschland zu einer erheblichen Zunahme von Präparaten aus dem Gebiet der Dermatologie geführt. Somit besteht auch ein zunehmender Bedarf an entsprechenden Weiterbildungsmöglichkeiten, insbesondere auf dem Gebiet der Neoplasien der Haut, die in diesem Themenheft besprochen werden sollen.

Melanozytäre Tumoren der Haut stellen eine besondere Herausforderung für den Kliniker und den Pathologen dar, nicht zuletzt durch die Existenz maligner Tumoren, die klinisch und histopathologisch eine benigne Läsion imitieren, oder z. B. die Gruppe der spitzoiden melanozytären Neoplasien, deren morphologi- sche Veränderungen oft fehlinterpretiert werden, was zu einer Übertherapie führen kann. Des Weiteren wurde in den letzten Jahren eine Reihe diagnostisch relevanter Formvarianten des malignen Melanoms beschrieben, deren Kenntnis sehr wichtig ist, um eine eventuelle Verwechslung mit benignen Veränderungen zu vermeiden.

Neben einem Beitrag zur problematischen Diagnose des dysplastischen Nävus wird das morphologische Spektrum des malignen Melanoms dargestellt. In den letzten Jahren konnte gezeigt werden, dass sowohl die spitzoiden melanozytären Tumoren als auch das maligne Melanom genetisch heterogene Gruppen melanozytärer Neoplasien repräsentieren, und die Bestimmung dieser molekularpathologischen Veränderungen besonders bei fortgeschrittenen melanozytären Neoplasien nicht nur diagnostische, sondern zunehmend auch eine wichtige therapeutische Bedeutung hat. So weisen mehr als die Hälfte der metastasierenden malignen Melanome Mutationen des BRAF-Gens auf (häufigste Mutation ist die V600E gefolgt von der V600K-Mutation), bei älteren Patienten mit Schleimhautmelanomen oder konjunktivalen malignen Melanomen wurden $c$-Kit-Mutationen und bei Uveamelanomen in einem Teil der Fälle GNAQ-Mutationen beobachtet.

Neue Therapeutika, die spezifische Inhibitoren repräsentieren und gezielt in Signaltransduktionswege und die immunregulatorische Funktion der Melanomzellen eingreifen (z. B. BRAF, NRAS, PD-(L)1), zeigen beim fortgeschrittenen malignen Melanom eine besondere Wirksamkeit, sind deshalb bereits zugelassen oder befinden sich in klinischen Studien. Durch diese zielgerichtete Therapie konnte das progressionsfreie Überleben von Patienten mit fortgeschrittenen malignen Melanomen verlängert werden; allerdings hat sich das mediane Gesamtüberleben aufgrund der Entwicklung von Resistenzen nur gering verbessert. Aus diesem Grunde wird derzeit die Kombination verschiedener Inhibitoren ( $\mathrm{z}$. B. BRAF- und MEK-Inhibitoren) getestet. Derzeit existieren noch keine zugelassenen Therapien für maligne Melanome mit KIT- und GNAS/GNAQ-Mutationen.

Durch diese aktuellen Entwicklungen wurden insbesondere die malignen melanozytären Tumoren zu einem wichtigen Entwicklungs- und Anwendungsbereich für die molekularpathologische Diagnostik. Diese Entwicklung hat Dermatologie und Pathologie noch enger zusammengeführt. In klinischen Studien, Tumorboards und auch der täglichen Diagnostik hat sich die Kooperation zwischen den Fachdisziplinen weiter vertieft, und ohne Prophet zu sein, kann man von einer weiteren Intensivierung der Zusammenarbeit ausgehen. Umgekehrt ist zu erwarten, dass analog zu anderen Tumorentitäten die umfassende molekulare Typisierung in Verbindung mit klinischen Daten auch die morphologische Diagnostik weiter modifizieren und präzisieren und vielfach zu einer integrierten molekularmorphologischen Typisierung und Nosologie führen wird.

Diagnostische Multi-Gentests werden derzeit auch beim malignen Melanom in die Diagnostik integriert. Diese Entwicklungen sind zukunftsweisend und sehr erfreulich und werden durch die Fachgesellschaften wissenschaftlich, aber auch durch gemeinsame Qualitätssicherungsmaßnahmen begleitet. Die morphologische Diagnostik der Hauttumoren, die unabhängig von allen molekularen Innovationen die solide Basis ist und bleibt, ist dadurch mehr gefordert aber auch zu- 
nehmend in den interdisziplinären Kontext eingebettet und vielfältiger geworden. Nicht zuletzt dadurch sind die Hauttumoren Paradigmen und auch Schrittmacher einer neuen Tumordiagnostik.

Bei der Diagnostik kutaner Pseudolymphome und primär kutaner maligner Lymphome spielen das klinische Bild und die zeitliche Entwicklung der Veränderungen eine sehr wichtige Rolle. Neben den etablierten Entitäten wie der Mycosis fungoides, den CD30-positiven lymphoproliferativen Erkrankungen, dem primär kutanen Keimzentrumslymphom, dem primär kutanen Marginalzonenlymphom vom MALT-Typ und dem primär kutanen diffusen großzelligen B-Zell-Lymphom wurde in den letzten Jahren eine Reihe z. T. sehr seltener, neuer Entitäten beschrieben, die teilweise durch einen sehr aggressiven klinischen Verlauf gekennzeichnet sind, weshalb ihre Kenntnis für den beurteilenden Pathologen umso wichtiger ist; sie werden in diesem Themenheft vorgestellt.

Prinzipiell können alle mesenchymalen Tumoren des Weichgewebes auch in der Haut auftreten, aber ihre Inzidenz sowie insbesondere ihre Prognose variieren deutlich. Darüber hinaus weisen einige Entitäten morphologische Unterschiede in Abhängigkeit von der Lokalisation auf. In diesem Themenheft werden mit den vaskulären und den sogenannten fibrohistiozytären Tumoren die häufigsten dermalen mesenchymalen Läsionen besprochen. Dabei wird zum einen auf seltene Formvarianten und auf die Differenzialdiagnosen des kutanen Angiosarkoms eingegangen. Zum anderen werden benigne und maligne sogenannte fibrohistiozytäre Neoplasien der Haut besprochen, die bezüglich der Liniendifferenzierung aber in der Mehrheit Tumoren mit einer fibroblastischen bzw. einer myofibroblastischen Differenzierung entsprechen.

Obwohl epitheliale Tumoren der Haut sehr häufig sind und in der Diagnostik keine Probleme bereiten, gibt es doch erhebliche konzeptionelle Probleme bei der Bewertung der früher als Präkanzerose eingeordneten Keratosis solaris. Des Weiteren hat es sich herausgestellt, dass das Keratoakanthom als eigenständige Entität und in seiner Beziehung zum gut dif- ferenzierten Plattenepithelkarzinom umstritten ist. Ergänzt werden diese Beiträge durch eine historische Betrachtung der Dermatohistologie in Deutschland und einen klinischen Beitrag, in dem nichtinvasive Verfahren zur Diagnostik von Neoplasien der Haut vorgestellt und diskutiert werden.

Ihre

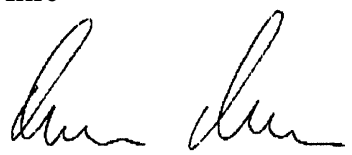

Prof. Dr. T. Mentzel

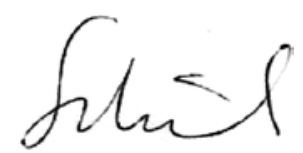

Prof. Dr. P. Schirmacher

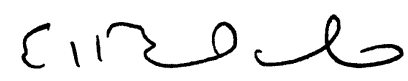

Prof. Dr. E. Bierhoff

\section{Korrespondenzadressen}

\section{Prof. Dr. T. Mentzel}

Dermatopathologie Bodensee

Siemensstraße 6/1, 88048 Friedrichshafen mentzel@dermpath.de

\section{Prof. Dr. P. Schirmacher}

Institut für Pathologie

Universitätsklinikum Heidelberg

Im Neuenheimer Feld 224

69120 Heidelberg

Peter.Schirmacher@med.uni-heidelberg.de

\section{Prof. Dr. E. Bierhoff}

Heinz-Werner-Seifert-Institut für

Dermatopathologie

Trierer Straße 70-72, 53115 Bonn

erhard.bierhoff@dermpath-bonn.de

\section{Einhaltung ethischer Richtlinien}

Interessenkonflikt. T. Mentzel, P. Schirmacher und E. Bierhoff geben an, dass kein Interessenkonflikt besteht.

\section{Nichtkleinzellige Lungen- karzinome}

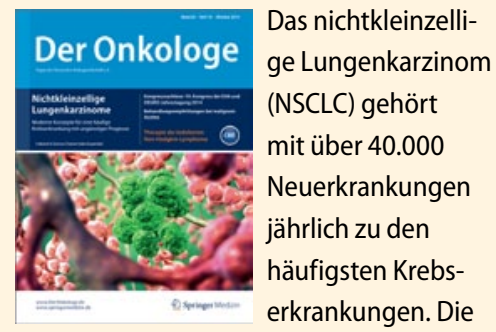

Prognose der Patienten ist nach wie vor nicht günstig; die Fünfjahresüberlebensrate liegt bei $15 \%$. Dennoch zeichnen sich Entwicklungen ab, die relevante Perspektiven aufzeigen. Ausgabe 10/2014 der Zeitschrift Der Onkologe widmet sich unter anderem molekularen Therapieansätzen, modernen Konzepten der lokoregionären Therapie sowie aktuellen Ansätzen zum Management der oligometastatischen Erkrankung.

Lesen Sie im Leitthemenheft mehr zu folgenden Themen:

- Pathologie als Gatekeeper

- Systemtherapie des NSCLC im metastasierten Stadium

- T1-Tumoren

- Stellenwert der Manschettenresektion am Bronchial- und Gefäßbaum der Lunge beim NSCLC

- Oligometastasiertes Lungenkarzinom

- Frühe palliative Intervention

Bestellen Sie diese Ausgabe zum Preis von 37,- EUR zzgl. Versandkosten bei Springer Customer Service Center Kundenservice Zeitschriften Haberstr. 7, 69126 Heidelberg Tel.: +49 6221-345-4303

Fax: +49 6221-345-4229

E-Mail: leserservice@springer.com

Suchen Sie noch mehr zum Thema? Mit e.Med, dem Online-Paket von Springer Medizin, können Sie schnell und komfortabel in über 600 medizinischen Fachzeitschriften recherchieren.

Weitere Infos unter springermedizin.de/ eMed. 